\title{
An Electronic Medical Device for Preventing and Improving the Assisted Ventilation of Intensive Care Unit Patients
}

\author{
R. Marani and A.G. Perri*
}

Dipartimento di Elettrotecnica ed Elettronica, Politecnico di Bari, Via E. Orabona 4, 70125, Bari, Italy

\begin{abstract}
The survival of patients submitted to Intensive Care Unit is often related to the mechanical ventilation, which makes up for serious respiratory insufficiencies but can also become a lethal health risk. To decrease these risks, the respiratory cycle has to be continuously monitored and a real-time suitable setting of the ventilator is required, in order to realize natural breathing similar conditions. In this paper we propose a microcontroller-based digital electronic device, designed and realized with reference to the most advanced and reliable electronic technologies, oriented to the monitoring of the respiratory cycle and the relevant ventilator setting. Moreover, the device allows the effective auscultation, the accurate processing and the detailed visualization (temporal and frequency graphs) of any lung sound. Then, it is suitable for the continuous real-time monitoring of breathing functions, resulting also very useful to diagnose respiratory pathologies.
\end{abstract}

Keywords: Electronic medical devices, respiratory cycle monitoring, analog-digital converter, microcontroller.

\section{INTRODUCTION}

The mechanical ventilation is one of most frequent activities in intensive care wards. However, it can give both the Acute Lung Injury (ALI) and the Acute Respiratory Distress Syndrome (ARDS) [1]. These pathologies, which sometimes can be lethal, are related to phlogistic processes due to damage of the lungs as a result of the mechanical stress induced by the artificial ventilation.

A single respiratory cycle consists of an inspiration and expiration. Inspiration requires contraction of the diaphragm and intercostal, parasternal and scalenus muscles which produces an increase of the lungs volume and, then, a pressure difference with the external environment.

Expiration is usually passive, resulting from elastic recoil of the lungs and does not require the assistance of the muscles.

The mechanical ventilation increases the lungs volume by exercising a suitable pressure on the airway gas. Due to the natural pulmonary elastance, an high pressure can induce serious damage, dysfunctions and consequent inflammatory reactions against the lungs.

To prevent these damages the respiratory cycle has to be continuously monitored and a real-time suitable setting of the ventilator is required, in order to realize natural breathing similar conditions.

Unfortunately, adequate electronic instruments for monitoring the respiratory cycle are missing.

The stethoscope auscultation, despite its advantages, has some well-known limitations consisting in some problems and criticality which have not been overcome yet, though

*Address correspondence to this author at the Dipartimento di Elettrotecnica ed Elettronica, Politecnico di Bari, Via E. Orabona 4, 70125, Bari, Italy; Tel: +390805963314, 390805963427; Fax: +390805963410;

E-mail: perri@poliba.it many changes and improvements have been being made to attain higher performances (traditional models now coexist with a fairly wide range of electronic stethoscopes).

Nevertheless, when using a stethoscope, the recognition and differentiation of lung sounds depend on nothing else but the individual's hearing abilities, experience and skillfulness. As a result the auscultation and interpretation of sounds performed by means of this instrument have a consistent subjective component.

This accounts for the need to go beyond the stethoscope (whether electronic or not) both to process the acquired signal by means of automatic calculation devices (thereby removing subjectivity) and to perform auscultation on a larger surface by placing sensors all over the thorax simultaneously.

Within a research program on the remote and noninvasive monitoring of the health, which has been developed at the Electronic Devices Laboratory of the first Faculty of Engineering at Bari Polytechnic, with the support of national university medical centres, a microcontroller-based digital electronic device has been designed and realized. This device, designed with reference to the most advanced and reliable electronic technologies, is oriented to the monitoring of the respiratory cycle and the relevant ventilator setting. The device provides medical specialists with a totally noninvasive high-engineering device able to detect and analyze the widest number of data for the monitoring of the respiratory system. It performs the monitoring and the analysis of respiratory health by the simple recording and evaluation of lung sounds $[2,3]$.

\section{RESPIRATORY CYCLE MONITORING METHOD AND DESIGN SPECIFICATIONS}

Methods adopted for the respiratory cycle monitoring can be distinguished into two types: static methods and dynamic methods. Static methods require ventilation interruption from 30 to 60 seconds, being very dangerous for the patients. 
One of the most important dynamic method is the Stress Index [4], which is based on the acquisition and following analysis of airway pressure values in constant flow ventilation condition (volume controlled). From the acquired curve we can derive a pulmonary stress index, which represents the extreme hypothesis of induced increase of lungs volume. A progressive decrease of the slope of the curve designates the alveolar involvement, while a progressive increase of the slope designates over-ventilation.

Therefore, the required parameters for monitoring the respiratory mechanics in the stress index method are flow, pressure and lungs volume.

The device acquires, in non-invasive manner, lowfrequency signals $\left(f_{\max }=200 \mathrm{~Hz}\right.$ ) from a pneumotacograph (flowmeter) and pressure sensors connected to patient's airway by plastic cannula.

The design specifications are high miniaturization level, noise immunity, low costs and chance for future expansions in terms of number of required sensors, implementation of Plug and Play sensors to simplify their use, configuration, easy and fast connection to any Personal Computer.

\section{DEVICE DESCRIPTION}

A block diagram of the proposed device is shown in Fig. (1).

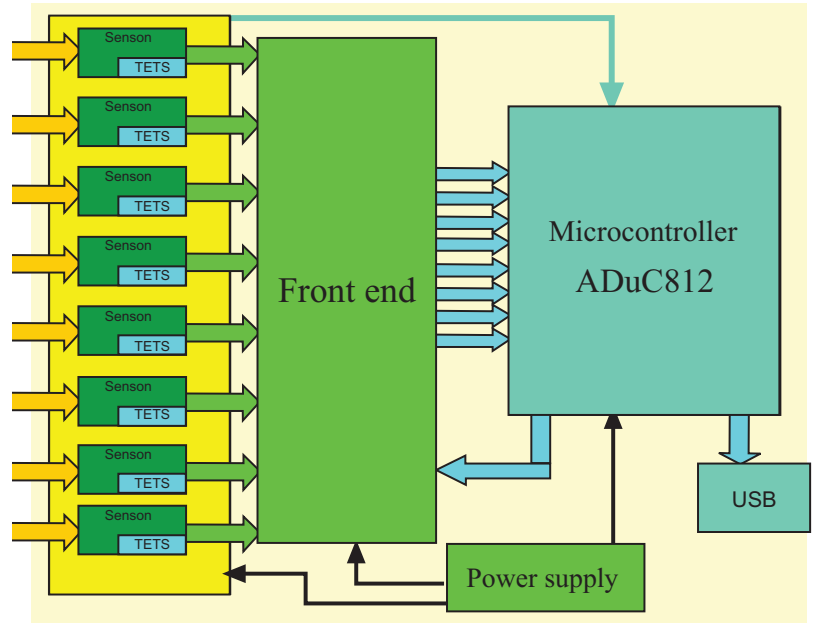

Fig. (1). Block diagram of the designed device.

The signals, coming from analog sensors, are suitably processed by the front-end and sampled at $1 \mathrm{KHz}$ frequency and, then, converted into digital format with 12-bit resolution, therefore guaranteeing high noise immunity.

The Front-End processes the signal to adapt the voltage values coming from the sensors to the input dynamic range (between $0 \mathrm{~V}$ and $2.5 \mathrm{~V}$ ) of the Analog-Digital Converter (ADC) included into the microcontroller.

Sensors can be unipolar (i.e. output voltages can be only positive or negative) or bipolar, where both positive and negative voltages are present. In both cases, the output signal amplitude can be greater than $2.5 \mathrm{~V}$, if each sensor includes an integrated amplifier.
The Front-End must diminish or amplifier the signal coming from each sensors, depending on its level and the input dynamic range of the ADC. If the signal is bipolar, a level shift is required to obtain a new signal greater than zero.

Since the signal processing depends on the sensor features, several shift-voltage values, each time determined by the microcontroller, have to be simultaneously produced [5]. Moreover, the gain of the amplifier has to be dynamically changed.

We have used only two programmable integrated circuits, controlled by a low-cost and high reliability (with particular reference to thermal drift phenomena) microcontroller, by implementing a device self-configuration procedure of the device to avoid any further maintenance work (such as calibration, front-end setting) by the user.

Microcontroller is required to program the Front-end functions, depending on sensor type, recognized by means of the implemented plug and play. The Three Wire Serial Interface Connections protocol has been used to establish a dialog between the Front-End and the microcontroller.

The Front-End process on a bipolar or unipolar sensor is shown in Figs. (2 and 3), respectively.

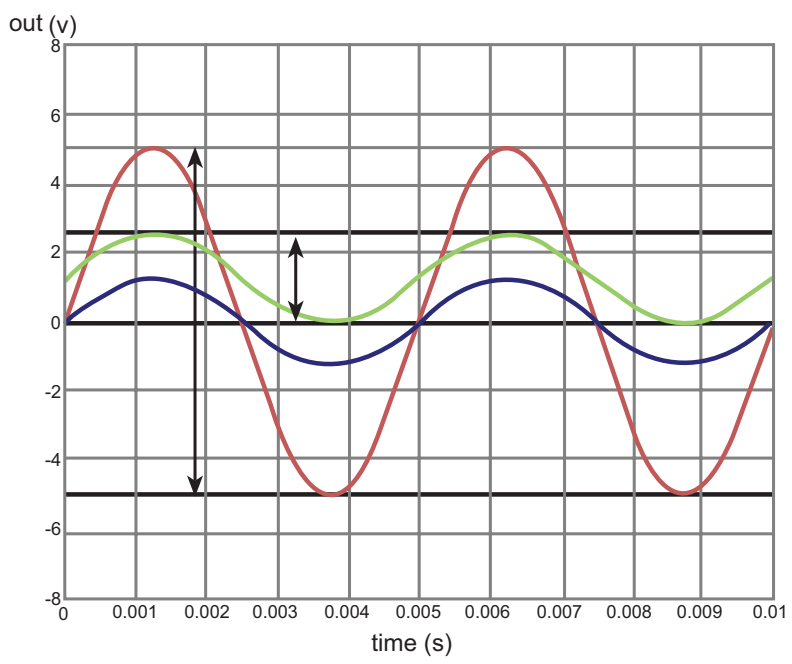

Fig. (2). Front-End process on a bipolar sensor with output range $(-5 \mathrm{~V},+5 \mathrm{~V})$.

We have used the ADuC812 Microcontroller, produced and distributed by Analog Devices, a low-cost device, which is very suitable to the design specifications.

A block diagram of the ADuC812 is shown in Fig. (4). The Microcontroller allows the data acquisition from 8 multiplexed channels, at a sample frequency up to $200 \mathrm{KHz}$, and can address up to $16 \mathrm{MB}$ of external data memory. The core is a 8052 compatible CPU, asynchronous output peripherals (UART) and synchronous serial SPI and $\mathrm{I}^{2} \mathrm{C}$.

The Sensor Plug and Play has been realized through implementation of IEEE standard P1451.4, with 1-wire system Communication Protocol.

Each sensor includes a transducer electronic data-sheet (TEDS), which stores the most significant informations rele- 


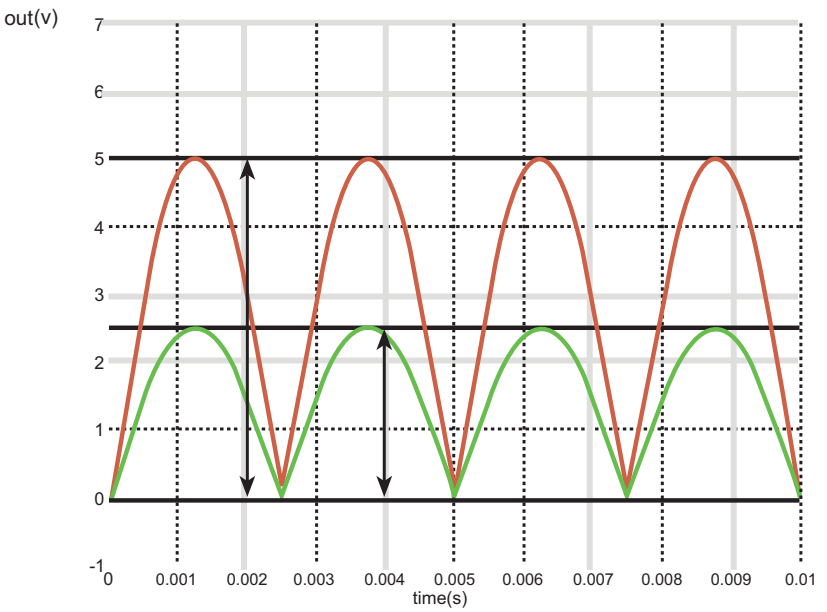

Fig. (3). Front-End process on an unipolar sensor with output range $(0 \mathrm{~V},+5 \mathrm{~V})$.

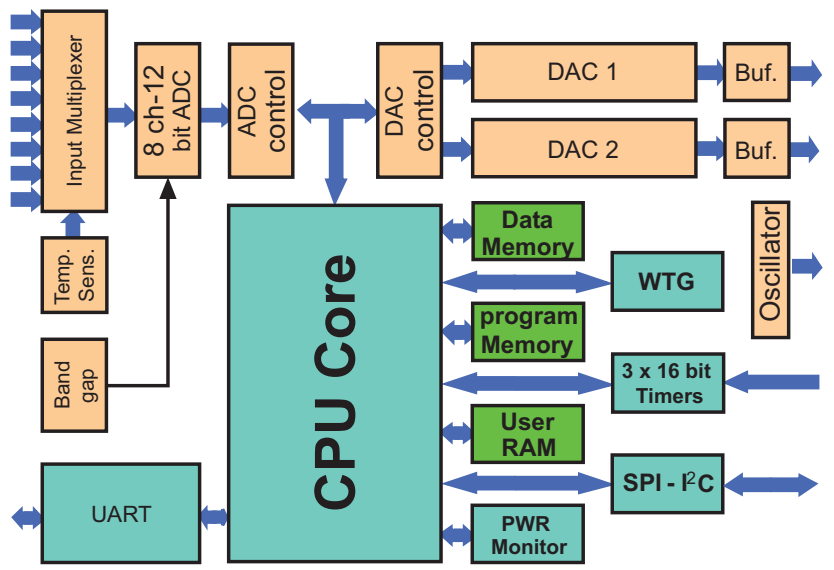

Fig. (4). Block diagram of the ADuC812 Microcontroller.

vant to the sensor type (manufacturer, offset, output range, etc).

Based on the stored data, microcontroller identifies the sensor and sets the Front-End device to suitably process the signal and perform the Analog-Digital conversion in very accurate manner. Each TEDS is a serial type ElectricallyErasable-Programmable Read Only Memory (EEPROM), connected to the microcontroller by only two wires.

A schematic of the proposed device is reported in Fig. (5), while the realized prototype is shown Fig. (6).

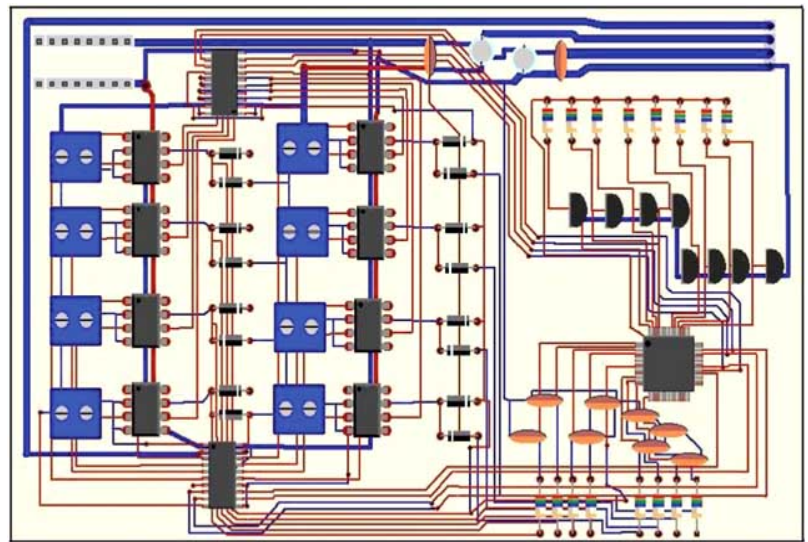

Fig. (5). Schematic of the designed device for monitoring the respiratory mechanics.

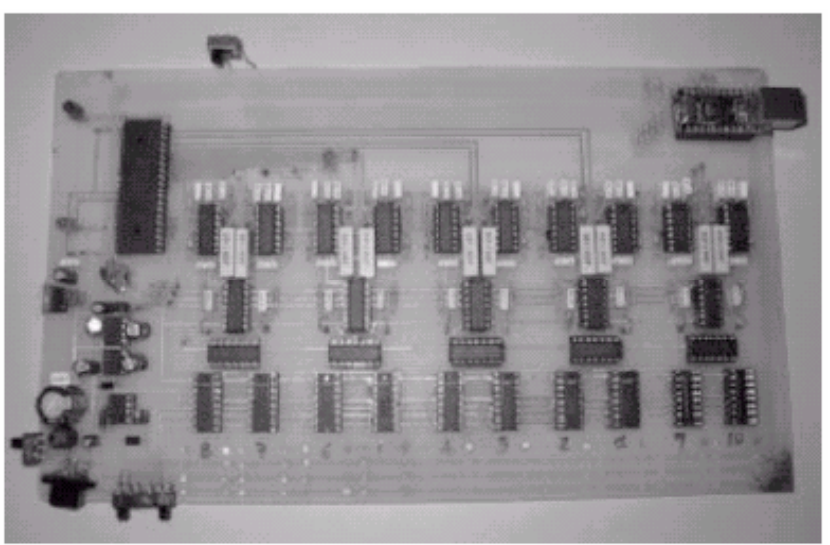

Fig. (6). The prototype: a double-sided printed circuit board.

The device is characterized by compactness and smallsize and performs the following operations: selfconfiguration, data-acquisition and conversion, data transfer to a Personal Computer and post-processing (such as ventilator setting).

All the data can be processed in real time, but an external memory support can be used to realize a data-bank accessible from any $\mathrm{PC}$.

\section{EXPERIMENTAL RESULTS}

The proposed device has been applied to plot the graph of a signal both while recording it (real time) and after saving it (see Fig. 7).
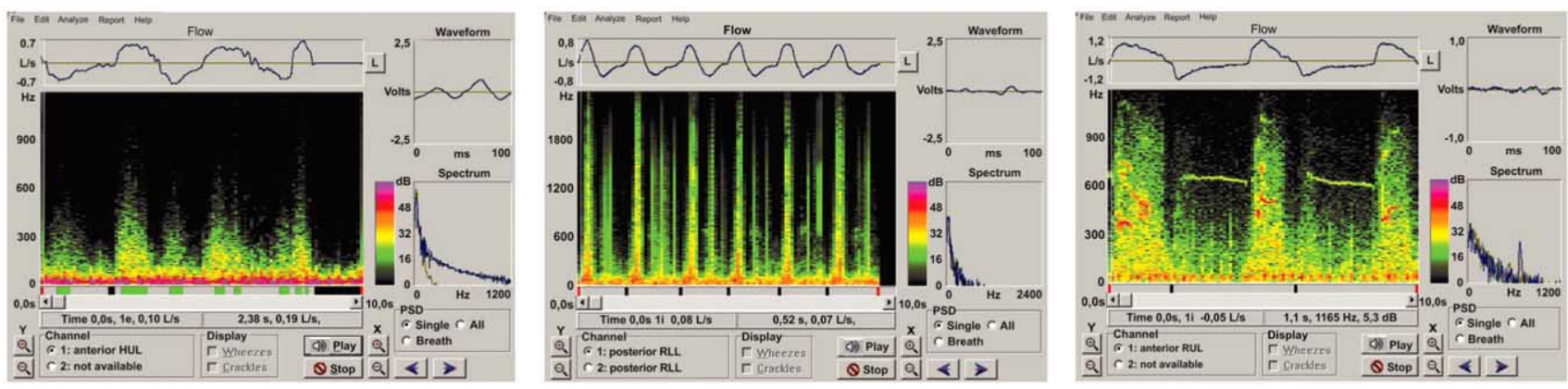

Fig. (7). Graphs of some lung sounds (respectively Normal, Crackle, Wheeze). 
Recent research has pointed out the effectiveness of the frequency analysis of lung sounds for the diagnosis of pathologies [4]. The experiment illustrated below shows that computerized tomography (CT) results perfectly match those of a simple frequency analysis of previously recorded lung sounds.

Many studies [6] have been carried out on the frequency analysis of lung sounds and researchers have set the threshold for the detection of pulmonary pathologies at $500 \mathrm{~Hz}$ (see Fig. 8).

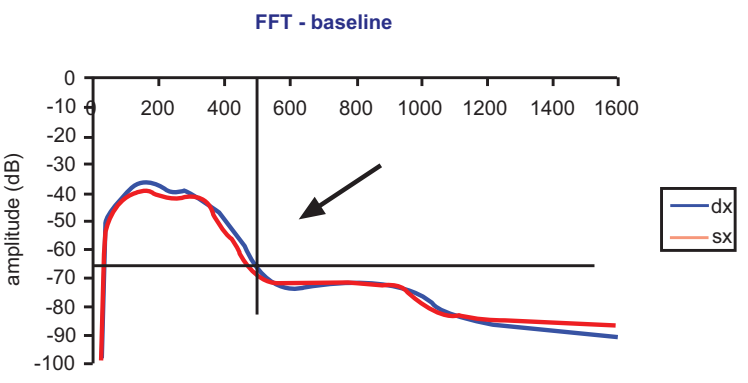

frequency $(\mathrm{Hz})$

Fig. (8). FFT - spectrum of a lung sound in a patient with no pulmonary pathologies.

Spectrum components over that threshold $(500 \mathrm{~Hz})$ may be indicative of pulmonary disease.

It is widely known that in patients treated with mechanical ventilation a gradual PEEP increase $($ PEEP $=$ positive end-expiratory pressure) results in a progressive re- expanding of alveoli which were previously collapsed due to a pathology.

The proposed device has been applied for the monitoring and the analysis of respiratory health by the simple recording and evaluation of lung sounds. The results, reported in Fig. (9), show how a gradual PEEP increase - from 5 to 20 - has effected a gradual reduction in lung damage (marked with a grey circle), thereby leading to improvement in the patient's respiratory health.

The CT results, shown in the first column, perfectly match those of the frequency analysis on the right.

Moreover, there are also research projects about pulmonary acoustic imaging for the diagnosis of respiratory diseases. In fact, the respiratory sounds contain mechanical and clinical pulmonary information. Many efforts have been devoted during the past decades to analysing, processing and visualising them [7].

We can now evaluate deterministic interpolating functions to generate surface respiratory acoustic thoracic images.

\section{CONCLUSIONS}

The main features of the proposed electronic medical device can be summarized in the following way:

- $\quad$ extreme simplicity of use;

- high miniaturization (available also with SMD components);

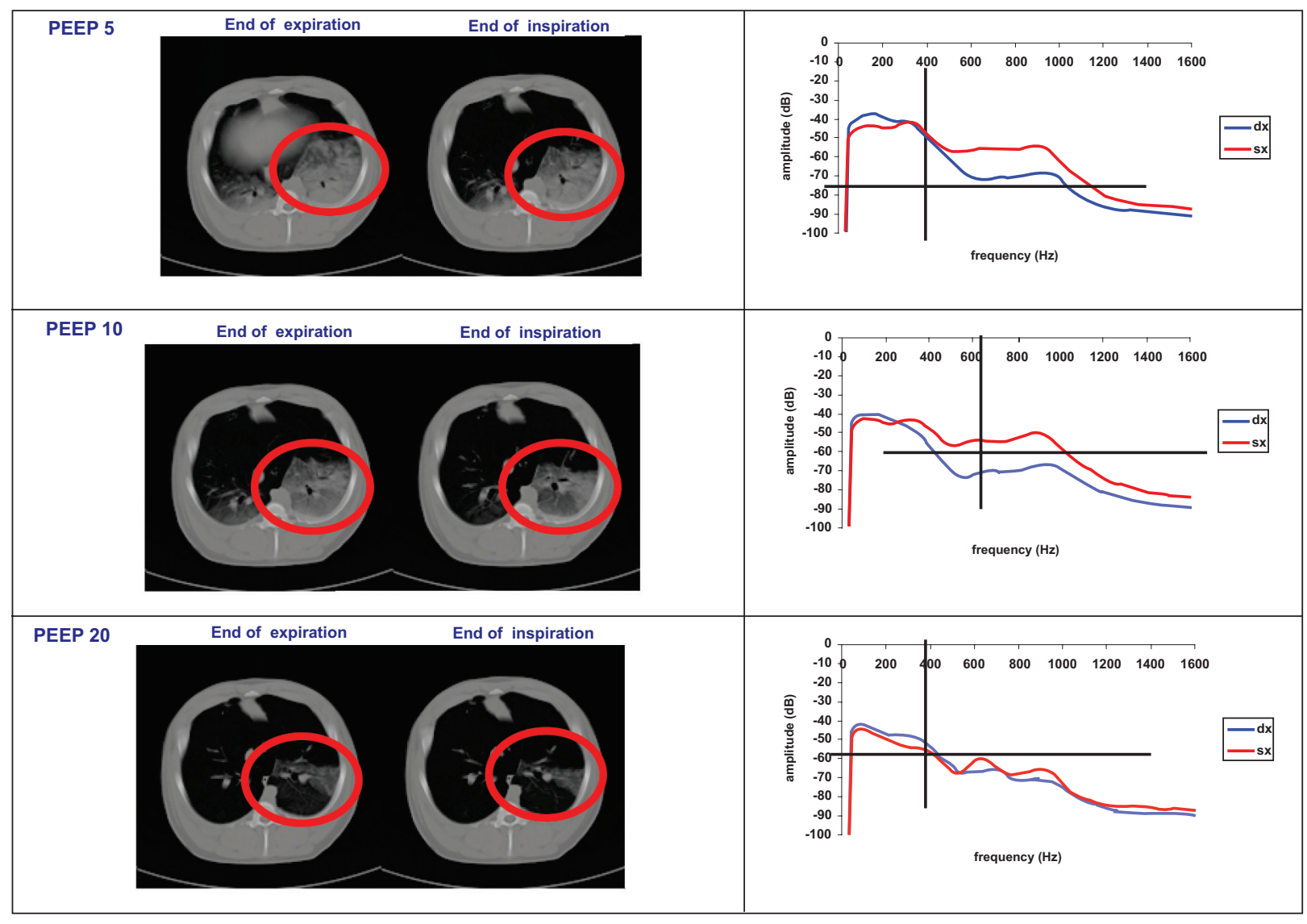

Fig. (9). Correlation between CT results and spectrum analysis of lung sounds. 
- USB interface;

- microcontroller based system;

- ten on board acquisition channels;

- $\quad$ simultaneous acquisition;

- $\quad$ specific channel for the pneumotachograph (optional);

- $\quad$ good resolution of the acquired signals (12 bit or 16 bit);

- high immunity from noise and environmental interference assured by signal digitalization;

- expandability for new applications and future improvements (through updating of the microcontroller firmware);

- $\quad$ plug and play module for the sensors;

- $\quad$ reasonable price of the end system.

The following options are also available:

- $\quad$ temporal graph of a breathing sound;

- frequency graph of a breathing sound;

- $\quad$ spectrogram of a breathing sound;

- temporal graph of the airflow (optional);

- measurement of both airflow and inspiratory/expiratory volume (optional).

Moreover the architecture is general and versatile and allows several signal processing in biological applications.
The proposed device has been applied for the monitoring and the analysis of respiratory health by the simple recording and evaluation of lung sounds.

We are now developing the firmware and the postprocessing software to optimize the device performance.

\section{ACKNOWLEDGEMENT}

The authors would like to thank Dr. A. Convertino for his assistance to realize the device prototype.

\section{REFERENCES}

[1] P. Rodriguez, M. Dojar, and L. Brochard, "Mechanical ventilation: changing concepts," Indian J. Crit. Care Med., vol. 9, pp. 235-43, 2005.

[2] A.R.A. Sovijärvi, J. Vandershoot, and J.E. Earis, "Standardization of computerized respiratory sound analysis," Eur. Respir. Rev., vol. 10, no. 77, p. 585, 2000.

[3] J.E. Earis, and B.M.G. Cheetham, "Future perspectives for respiratory sound research," Eur. Respir. Rev., vol. 10, pp. 641-77, 2000.

[4] S. Grasso, L. Mascia, T. Trotta, M. Di Fonzio, M. Ribezzi, F. Puntillo, G. Ancona, F. Bruno, T. Fiore, F. Giunta, A. Slutsky, M. Ranieri, "Dynamic airway pressure/time curve analysis to realize lung protective ventilatory strategy in ARDS patients," Intensive Care Med., 2000. http://www.euroanesthesia.org/education/rc_gothenburg/12rc8.HTML

[5] M.V. Kirianaki, "Data Acquisition Signal Processing for Smart Sensors," USA, John Wiley \& sons, vol. 178, 2002.

[6] A. Vena, G. Perchiazzi, G. M. Insolera, R. Giuliani, T. Fiore, "Computer analysis of acoustic respiratory signals, Modelling biomedical signals," Singapore, World Scientific Publishing, vol. $60,2002$.

[7] M. Kompis, H. Pasterkamp, G. R. Wodicka, "Acoustic imaging of the human chest," Chest, vol. 120, pp. 1309-1321, 2001.

(C) Marani and Perri; Licensee Bentham Open.

This is an open access article licensed under the terms of the Creative Commons Attribution Non-Commercial License (http://creativecommons.org/licenses/by-nc/3.0/) which permits unrestricted, non-commercial use, distribution and reproduction in any medium, provided the work is properly cited. 